Corrigendum

\title{
Corrigendum to "Effects of Combined Use of Ultrasonic Bone Scalpel and Hemostatic Matrix on Perioperative Blood Loss and Surgical Duration in Degenerative Thoracolumbar Spine Surgery"
}

\author{
Hou-Tsung Chen ${ }^{1},{ }^{1}$ Chieh-Cheng Hsu, ${ }^{1}$ Meng-Lin Lu, ${ }^{1}$ Sung-Hsiung Chen, ${ }^{1}$ \\ Jing-Miao Chen, ${ }^{1}$ and Re-Wen Wu (D) ${ }^{1,2}$ \\ ${ }^{1}$ Department of Orthopaedic Surgery, Kaohsiung Chang Gung Memorial Hospital, Kaohsiung, Taiwan \\ ${ }^{2}$ Graduate Institute of Clinical Medical Sciences, Chang Gung University, Taiwan \\ Correspondence should be addressed to Re-Wen Wu; ray4595@gmail.com
}

Received 17 July 2019; Accepted 17 July 2019; Published 4 August 2019

Copyright (C) 2019 Hou-Tsung Chen et al. This is an open access article distributed under the Creative Commons Attribution License, which permits unrestricted use, distribution, and reproduction in any medium, provided the original work is properly cited.

In the article titled "Effects of Combined Use of Ultrasonic Bone Scalpel and Hemostatic Matrix on Perioperative Blood Loss and Surgical Duration in Degenerative Thoracolumbar Spine Surgery" [1], the IRB number was given incorrectly as (IRB201700086B0). The correct number should be (IRB201600834B0). Therefore, the second sentence in subsection "2.1. Patients" should be updated as follows:

The study was conducted with a waiver of patient consent and was approved by the Institutional Review Board of our hospital (IRB201600834B0).

\section{References}

[1] H. Chen, C. Hsu, M. Lu, S. Chen, J. Chen, and R. Wu, "Effects of combined use of ultrasonic bone scalpel and hemostatic matrix on perioperative blood loss and surgical duration in degenerative thoracolumbar spine surgery," BioMed Research International, vol. 2019, Article ID 6286258, 7 pages, 2019. 


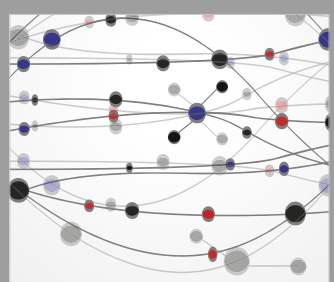

The Scientific World Journal
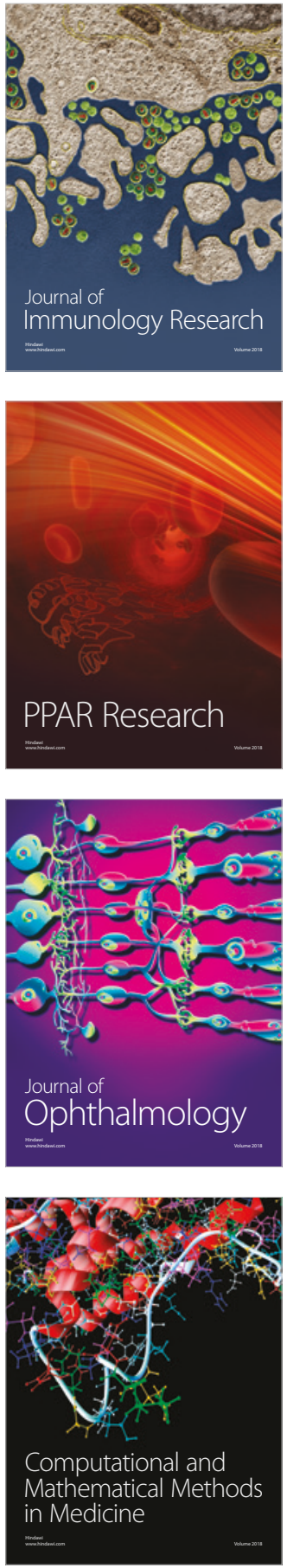

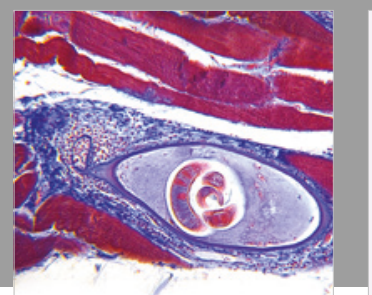

Gastroenterology Research and Practice

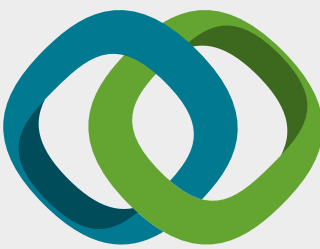

\section{Hindawi}

Submit your manuscripts at

www.hindawi.com
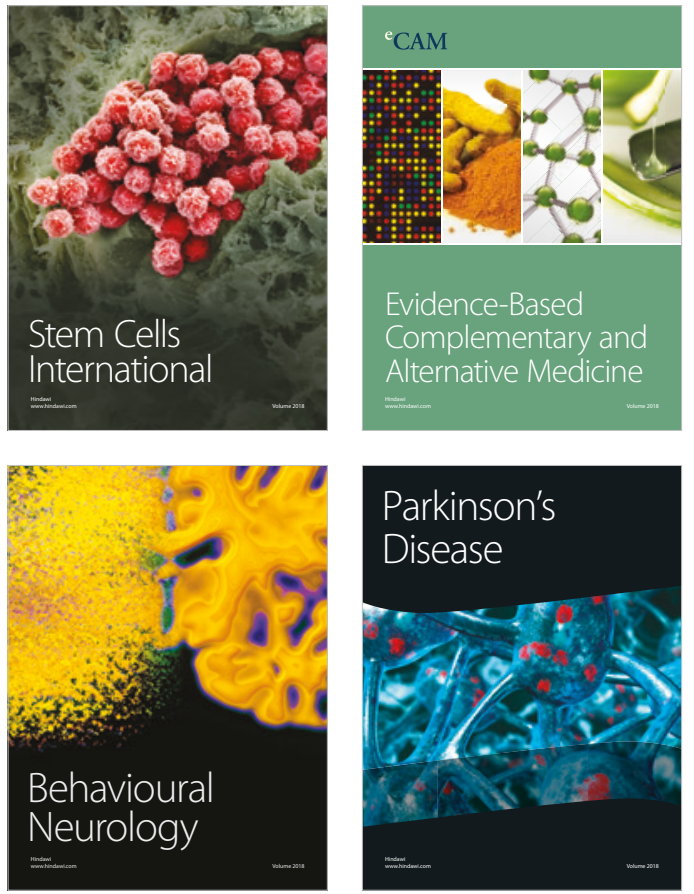

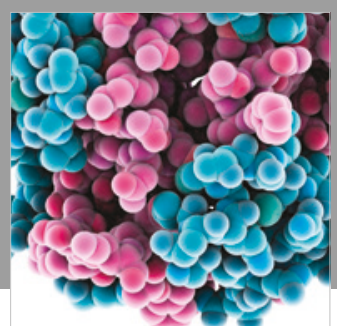

ournal of

Diabetes Research

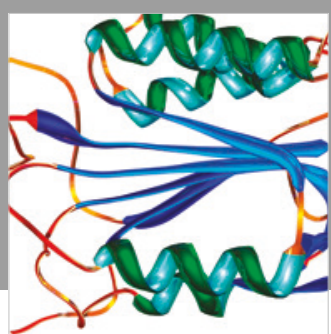

Disease Markers
